\title{
Evaluation of Type-A Endonucleases for the Quantitative Analysis of DNA Damage due to Exposure to Acetaldehyde Using Capillary Electrophoresis
}

\author{
Yukihiro EsaKa, ${ }^{* * * *}$ Kenji Hisato, $*$ Takuhei Yamamoto,* Hiroya Murakami, ${ }^{* * *}$ and \\ Bunji UNO**** \\ *Gifu Pharmaceutical University, 1-25-4 Daigaku-nishi, Gifu 501-1196, Japan \\ **United Graduate School of Drug Discovery and Medical Information Sciences, Gifu University, 1-1 Yanaido, \\ Gifu 501-1194, Japan \\ ***Department of Applied Chemistry, Aichi Institute of Technology, 1247 Yachigusa, Yakusa-cho, Toyota 470-0392, \\ Japan
}

\begin{abstract}
The substrate selectivities of three endonucleases were studied quantitatively using capillary zone electrophoresis to find one giving $N^{2}$-ethyl(Et)-2'-deoxyguanosine-5'-monophosphate (5'-dGMP) and cyclic 1, $N^{2}$-propano(CPr)-5'-dGMP from DNAs damaged by acetaldehyde (AA). Six $2^{\prime}$-deoxyribonucleoside- $5^{\prime}$-monophosphates to be quantified in the hydrolysis solutions of DNAs, namely, Et-5'-dGMP, CPr-5'-dGMP, and four authentic ones, were completely separated using a $100 \mathrm{mM}$ borate running buffer solution having an optimized $\mathrm{pH}$ of 9.67. Using the present method, nuclease reactions of nuclease S1 (NS1), nuclease P1 (NP1), and nuclease Bal 31 to 2'-deoxyribonucleoside-5'-monophosphates from damaged Calf thymus (CT-) DNAs were monitored. The CT-DNAs were prepared by treatment with AA to generate Et-guanine or CPr-guanine internally. Bal 31 hydrolyzed the damaged CT-DNAs to yield Et-5'-dGMP and CPr-5'-dGMP quantitatively. The two 5'-dGMP adducts were not detected in the hydrolysis solutions using NS1 or NP1. Bal 31 can be a suitable nuclease for analyzing DNA damages caused by AA.
\end{abstract}

Keywords Endonuclease, DNA damage, acetaldehyde, $N^{2}$-ethyl-5'-dGMP, cyclic 1, $N^{2}$-propano- $5^{\prime}$-dGMP, capillary zone electrophoresis

(Received February 26, 2018; Accepted March 27, 2018; Published August 10, 2018 )

\begin{abstract}
Damages of DNAs in base moieties are known to be a major pathway to carcinogenesis. Such damages occur because of attacks from chemicals or exposure to ultraviolet light and radioactive rays. The existing frequency and structure of the damaged bases in personal DNA reflect the owners' habits, such as smoking and drinking, as well as living and labor environments, genetic backgrounds, and aging situations. Therefore, the damaged DNA bases can become cancer risk markers. ${ }^{1-3}$ Generally, the damaged base moieties in DNAs are detected as the corresponding monomer forms (bases, deoxyribonucleosides, or deoxyribonucleoside monophosphates). ${ }^{4-12}$ Therefore, it is important to investigate the substrate selectivity of nucleases, which are usually used in pretreatments of DNAs as mid or final steps for analyzing DNA damages to obtain object damaged monomers from DNAs. Native nucleases are expected to prefer the authentic base moieties to damaged ones in DNAs as substrates. ${ }^{13}$ If nucleases are used, which possess preferential selectivities for the authentic base moieties in DNAs, the corresponding damaged nucleotide monomers would not be given quantitatively, or even at all. The adequacy of nucleases in substrate selectivity toward object damaged base moieties in DNAs has to be confirmed before the nucleases are
\end{abstract}

$\dagger$ To whom correspondence should be addressed.

E-mail: esaka@gifu-pu.ac.jp used for the hydration of DNAs.

In this work, three kinds of commercially available type-A endonucleases, working on heat-denatured DNAs to generate the constituent 2'-deoxyribonucleoside-5'-monophosphates, were evaluated for determining $N^{2}$-ethyl(Et)-guanine (Gua) and cyclic $1, N^{2}$-propano(CPr)-Gua in DNAs, which were detected as Et-2'-deoxyriboguanosine- $5^{\prime}$-monophosphate $\left(5^{\prime}\right.$-dGMP) and CPr-5'-dGMP here. Both Et-Gua and CPr-Gua are adducts of Gua by acetaldehyde (AA) (Fig. 1) and some part of the two adducts existing in DNAs of drinkers is considered as those derived from drinking. ${ }^{4,11,14}$ In a previous report, Vaca et al. demonstrated that the DNAs of alcoholic patients included a<smiles>CCNc1nc2[nH]cnc2c(=O)[nH]1</smiles>

(A)<smiles>CC1CC(O)n2c(nc3[nH]cnc3c2=O)N1</smiles>

(B)
Fig. 1 Structures of $N^{2}$-ethyl-guanine (Et-Gua) (A) and cyclic 1, $N^{2}$ propano-guanine (CPr-Gua) (B) as adducts by acetaldehyde. 
ca. 10-times larger amount of Et-Gua compared with control groups. ${ }^{11}$ On the other hand, our early work confirmed the generation of CPr-Gua in the DNA of HL-50 cells cultured in the present of AA. ${ }^{4}$ The two AA adducts of Gua are major candidates of biomarkers that express personal risks of cancer promotion caused by drinking. The cancer-promoting effects of drinking have attracted attention in recent years, which is considered to be attributed to AA as the first metabolite of ethanol. In fact, it has been confirmed epidemiologically that persons who have genetic deficiencies of AA dehydrogenase type 2, causing higher blood concentrations of AA compared with controls, possess more remarkable risks for carcinogenesis than the controls. ${ }^{15}$ As a result, International Agency for Research on Cancer (IARC) classifies ethanol in alcoholic beverages Group 1, meaning "carcinogenic to humans". ${ }^{16}$ Furthermore, AA also comes into bodies from smoking and automobile exhaust gas. ${ }^{17-19}$ From the viewpoint of preventive healthcare, the existing frequencies of the two AA adducts of Gua in personal DNAs should be monitored in periodic health checkups in the future.

Capillary electrophoresis (CE) has been successfully employed in the separation of nucleic acids. In particular, small nucleotides, such as mono-, di-, and triphosphates, can be separated well using the simple capillary zone electrophoresis (CZE) mode. ${ }^{20-22}$ Furthermore, with the addition of SDS micelles to running solutions of $\mathrm{CE}$, nucleobases and nucleosides can also be analyzed simultaneously by the additional micellar electrokinetic chromatography (MEKC) mode. ${ }^{23}$ Besides, nucleotides as phosphate compounds often absorb residual metal ions in stationary phases and stainless-steel parts in the flow channels of HPLC to retard the quantitative analysis of the nucleotides. ${ }^{24}$ In contrast, CE is performed in hollow fused silica tubes with on-column detection practically without contact with metal residues and parts. CE also consumes a very small amount of sample solutions using direct injection to capillaries, which is important in the analysis of commonly scarce biological samples. The evaluation of nucleases was also performed at low consumptions of enzymes and DNAs using CE in this work.

The aim of this work is to find a nuclease that hydrolyzes DNAs quantitatively to Et-5'-dGMP and CPr-5'-dGMP as candidates of cancer risk makers related to drinking as well as 5'-dAMP, 5'-dCMP, 5'-dGMP, and 5'-dTMP. It was necessary to study the substrate selectivities of nucleases. ${ }^{13}$ Et- $5^{\prime}$-dGMP, CPr-5'-dGMP, and the four authentic $2^{\prime}$-deoxyribonucleoside $5^{\prime}$-monophosphates were separated completely in the present $\mathrm{CE}$ system. The separation conditions were optimized mainly in terms of the $\mathrm{pH}$ values of the running solutions, while taking account of the $\mathrm{p} K_{\mathrm{a}}$ values of their nucleobase moieties. The optimized method was employed to evaluate the substrate selectivities of three type A endonucleases.

\section{Experimental}

\section{Reagents and chemicals}

Gua and 2'-deoxythymidine-5'-monophosphate (5'-dTMP) were purchased from Kohjin Life Science (Tokyo, Japan). 5'-dGMP, 2'-deoxyadenosine-5'-monophosphate (5'-dAMP), $2^{\prime}$-deoxycitidine- $5^{\prime}$-monophosphate $\quad\left(5^{\prime}\right.$-dCMP), cytidine- $5^{\prime}$ monophosphate (5'-CMP) as an internal standard (IS), and Calf thymus DNA (CT-DNA) were obtained from Sigma Aldrich Japan (Tokyo, Japan). Standards of CPr-Gua, Et-Gua, CPr-5'dGMP, and Et-5'-dGMP were synthesized according to previous reports. ${ }^{25,26}$ Nuclease P1 (NP1) was purchased from Wako Pure Chemical Industries (Osaka, Japan). Nuclease S1 (NS1) and nuclease Bal 31 (Bal 31) were purchased from Takara Bio Inc. (Shiga, Japan). Other chemicals were of analytical grade.

\section{Apparatus}

All electrophoretic measurements were performed on an Otsuka Electronics Capi-3200Q system (Shiga, Japan) equipped with bare fused-silica capillary tubing (Polymicro Technologies, Phoenix, USA) of ID $=0.050 \mathrm{~mm}$ and $\mathrm{OD}=0.375 \mathrm{~mm}$. The total length and effective length of the tubing were 572 and $450 \mathrm{~mm}$, respectively. An Iwaki Model ALB-301 bath (Shizuoka, Japan) was used as the incubator for enzymatic reactions. The concentrations of nucleotides were measured using a Shimadzu UV-3600 UV-vis spectrophotometer (Kyoto, Japan) equipped with a $1 \times 1 \mathrm{~cm}$ quartz cell.

\section{Procedure}

Preparation of damaged CT-DNAs as test samples for nuclease reaction analysis. The commercial CT-DNA was treated with AA to partially modify the amount of Gua in the DNA to form CPr-Gua or Et-Gua, as follows: ${ }^{14} 2 \mathrm{mg}$ of CT-DNA was dissolved in $900 \mu \mathrm{L}$ of a $0.1 \mathrm{M}$ phosphate buffer ( $\mathrm{pH}$ 7.2). The solution was heated at $95^{\circ} \mathrm{C}$ for $10 \mathrm{~min}$ and cooled immediately in ice to denature the DNAs to the single-strand form. For the formation of CPr-Gua in DNA, $4 \mathrm{mg}$ of arginine was dissolved in the denatured CT-DNA solution, followed by the addition of $100 \mu \mathrm{L} \mathrm{AA}$. Then, the mixed solution was incubated at $37^{\circ} \mathrm{C}$ for $6 \mathrm{~h}$. On the other hand, Et-Gua was produced in the CTDNA upon the addition of $100 \mu \mathrm{L} \mathrm{AA}$ and $20 \mathrm{mg} \mathrm{NaBH}{ }_{3} \mathrm{CN}$ to the denatured CT-DNA solution. Then, the mixed solution was incubated at $50^{\circ} \mathrm{C}$ for $2 \mathrm{~h}$. After the reactions finished, both reaction solutions were dialyzed for $12 \mathrm{~h}$ using a cellulose membrane. Inner solutions were stocked as the corresponding damaged DNA solutions at $4^{\circ} \mathrm{C}$.

Nuclease reactions. Native CT-DNA and the two AA-treated CT-DNAs, including Et-Gua or CPr-Gua, were hydrolyzed using three commercially available nucleases (NS1, NP1, or Bal 31 ), respectively, to give 2 -deoxynucleoside- $5^{\prime}$-monophosphates, as follows: $50 \mu \mathrm{g}$ of each CT-DNA was dissolved in $50 \mu \mathrm{L}$ of MilliQ water. Then, the solution was heated at $95^{\circ} \mathrm{C}$ for $5 \mathrm{~min}$, followed by cooling in ice to denature the DNA. For NP1 reactions, a $3-\mu \mathrm{L}$ NP1 solution $(0.25$ units $/ \mu \mathrm{L})$ and a $40 \mathrm{mM}$ acetate buffer ( $\mathrm{pH} 5.3$ ) containing $200 \mathrm{mM} \mathrm{ZnCl}_{2}$ were added to the denatured DNA solution. ${ }^{27}$ For NS1 reactions, a 5- $\mu \mathrm{L}$ NS1 solution (180 units/ $\mu \mathrm{L})$ and an accompanying buffer solution $(45 \mu \mathrm{L}, 30 \mathrm{mM}$ acetate buffer $(\mathrm{pH} 4.6)$ containing $10 \mathrm{mM}$ $\mathrm{ZnSO}_{4}$ ) were added in the denatured DNA solution. In addition, for Bal 31 reactions, a $1-\mu \mathrm{L}$ Bal 31 solution (2 units/ $\mu \mathrm{L})$ and an accompanying buffer solution $(49 \mu \mathrm{L}, 20 \mathrm{mM}$ Tris- $\mathrm{HCl}$ buffer (pH 8.0) containing $600 \mathrm{mM} \mathrm{NaCl}, 12 \mathrm{mM} \mathrm{CaCl}, 12 \mathrm{mM}$ $\mathrm{MgCl}_{2}$, and $1 \mathrm{mM}$ EDTA) were added to the denatured DNA solution. The whole incubation time was $1 \mathrm{~h}$.

The time courses of the yields for $5^{\prime}$-dAMP, $5^{\prime}$-dCMP, $5^{\prime}$-dGMP, and 5'-dTMP, which were generated in reactions of native CT-DNA samples by NP1, NS1, and Bal 31, were monitored from 15 to $120 \mathrm{~min}$. It was confirmed that all of the reactions were finished within $1 \mathrm{~h}$ under the present conditions [data not shown]. The yields of the reactions of native CT-DNA samples by the three nucleases were estimated to be $c a$. $80 \%$ based on the amount of the resultant $5^{\prime}$-dGMP. The increase in the amount of nucleases did not further increase the yields. These yields might be underestimated because of the low purity of the commercial CT-DNA (The value of $A_{260} / A_{280}$ for the present CT-DNA sample was 1.7 ). Besides, there were possibly some inevitable errors in the DNA concentrations calculated using the equation, i.e., $A_{260} \times 50 \mu \mathrm{g}=\mathrm{DNA}$ concentration 
$(\mu \mathrm{g} / \mathrm{mL}){ }^{28} A_{260}$ and $A_{280}$ were the absorbance at 260 and $280 \mathrm{~nm}$ with $1 \mathrm{~cm}$ length of light pass, respectively. The reactions were considered to be finished under the present conditions.

CE measurements. First, $100 \mathrm{mM}$ borate buffers were prepared from boric acid solutions. Their $\mathrm{pH}$ values were adjusted using a $1 \mathrm{~mol} / \mathrm{L} \mathrm{NaOH}$ solution. The buffers at each $\mathrm{pH}$ value added by $80 \mathrm{mM}$ SDS were used as the running solution for CE measurements. The detection wavelength was set at $254 \mathrm{~nm}$. The potential for the separation was applied at $19 \mathrm{kV}$. Sample solutions were injected hydrodynamically at a height of $25 \mathrm{~mm}$ for $300 \mathrm{~s}$. The operation temperature was $25^{\circ} \mathrm{C}$. Each peak was assigned by spiking the standard solutions of the seven nucleotides, if necessary. To facilitate the peak assignment, electropherograms of hydrolysis solutions were restructured using the electrophoretic mobility $\left(m_{\mathrm{ep}}\right)$ as $\mathrm{X}$-axis, according to the following equation: $l / t=1 / t_{\mathrm{eof}}+m_{\mathrm{ep}} L / P$, where $l, L, t, t_{\mathrm{eof}}$, and $P$ denote the effective length $[\mathrm{m}]$, total length of the capillary $[\mathrm{m}]$, migration time [min], migration time of the electroosmotic flow (EOF) marker [m], and applied potential [V], respectively. ${ }^{29}$

Plots of the relative peak areas for the six nucleotides using CMP as an internal standard (IS) versus the concentrations of the sample solutions were made as calibration curves for quantifying the six nucleotides under the optimized conditions. The $5^{\prime}$-CMP concentration in the sample solutions was fixed as $0.10 \mathrm{mM}$ in all measurements. Standard solutions of the four authentic nucleotides were prepared using the published $A_{260}$ data, i.e., $15.3 \times 10^{3}, 7.4 \times 10^{3}, 11.5 \times 10^{3}$, and $9.3 \times 10^{3} \mathrm{M}^{-1} \mathrm{~cm}^{-1}$ for $5^{\prime}$-dAMP, $5^{\prime}$-dCMP, $5^{\prime}$-dGMP, and $5^{\prime}$-dTMP as the corresponding moieties in a single-strand DNA, respectively. ${ }^{27}$ Nucleotide reagents generally have strong moisture absorbency as well as exist with counter ions and crystal water molecules of various composition ratios. This situation causes large errors in quantitation using direct weighing. The molar absorbance coefficients of Et-5'-dGMP and CPr-5'-dGMP are not known. The concentrations of their standard solutions were thus estimated as follows: $10 \mu \mathrm{L}$ of $1 \mathrm{M} \mathrm{HCl}$ aq. was added in $90 \mu \mathrm{L}$ of a stock solution of Et-5'-dGMP or CPr-5'-dGMP ( ca. 10 $\left.5^{-4} \mathrm{M}\right)$. Each mixed solution was incubated at $70^{\circ} \mathrm{C}$ for $1 \mathrm{~h}$. It was confirmed that both Et-5'-dGMP and CPr-5'-dGMP in the mixed solutions were converted quantitatively to Et-Gua and CPr-Gua, respectively, using the present $\mathrm{CE}$ method. The additional MEKC mode worked to separate the two base forms. The concentrations of the stock solutions of the two damaged nucleotides were estimated as being the resultant amounts of Et-Gua and CPr-Gua in the acid decomposed solutions, which were determined using the synthesized standards of Et-Gua and CPr-Gua.

\section{Results and Discussion}

CE separation of the six 2'deoxyribonucleoside-5'monophosphates CZE enables us to separate nucleotides having good selectivities by optimizing the $\mathrm{pH}$ of running solutions to control the proton dissociation of the functional groups of some base moieties. ${ }^{30}$ The electrophoretic mobility of some nucleotides possessing acidic $\mathrm{NH}$ groups adjacent to carbonyl groups of the bases, like those of thymine $\left(\mathrm{p} K_{\mathrm{a}}=9.8\right)$, guanine $\left(\mathrm{p} K_{\mathrm{a}}=9.2\right)$ and their related one increases markedly, compared with the other ones without acidic groups, when the $\mathrm{pH}$ is increased in the range covered by the buffer ability of borate $\left(\mathrm{p} K_{\mathrm{a}}=9.2\right)$ as the buffer component of the present running solutions. Also, as expected in the present case, the separation of the six nucleotides depended on the $\mathrm{pH}$ values of the running solutions from $\mathrm{pH} 9.0$

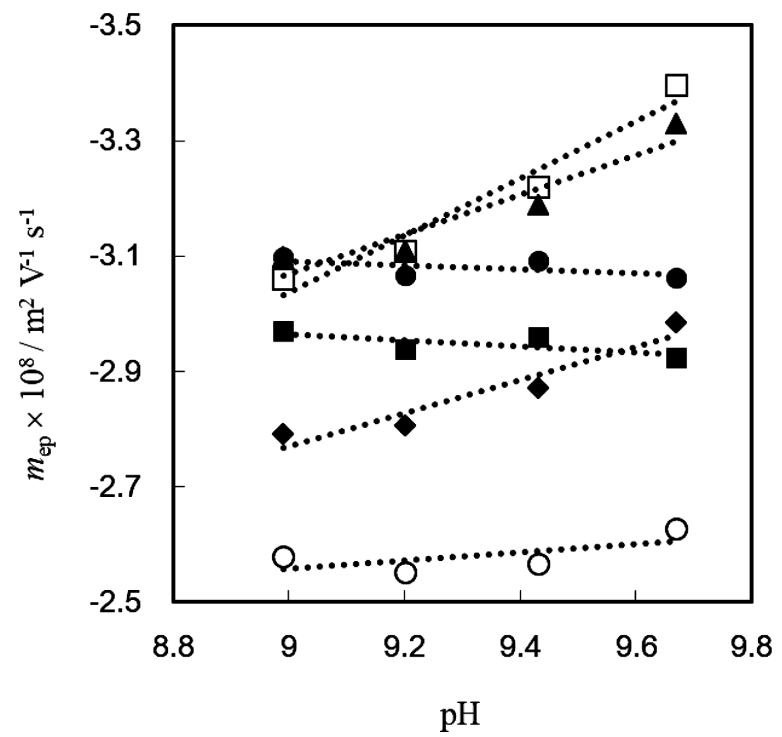

Fig. $2 \mathrm{pH}$ dependence of the electrophoretic mobilities $\left(m_{\mathrm{ep}}\right)$ of the six $2^{\prime}$-deoxyribonucleoside $5^{\prime}$-monophosphates. CE conditions: same as those in Fig. 3, except for pH. Symbols:, $5^{\prime}$-dAMP; $\boldsymbol{\Delta}, 5^{\prime}$-dTMP;

口, 5'-dCMP; $\diamond, 5^{\prime}$-dGMP; $\bigcirc$, CPr-5'-dGMP; $\square$, Et-5'-dGMP.

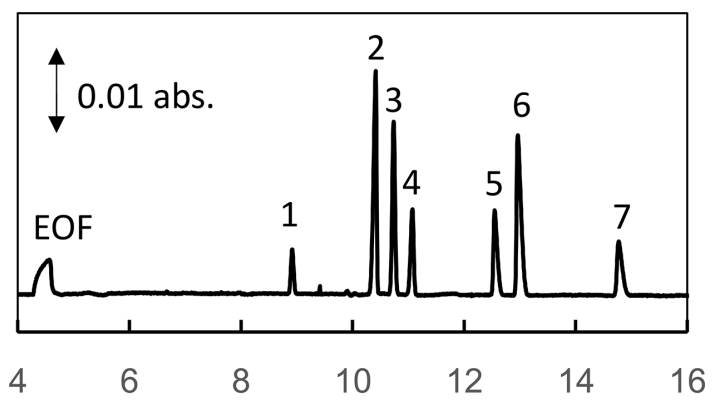

Migration time / min

Fig. 3 CZE separation of the seven nucleotides including 5'-CMP as IS under the optimized conditions. CE conditions: running solution, $100 \mathrm{mM}$ borate buffer solution of $\mathrm{pH} 9.67$ containing $80 \mathrm{mM}$ SDS; capillary, bare fused silica tubing, $0.050 \mathrm{~mm}$ i.d. $(0.375 \mathrm{~mm}$ o.d.), total length of $572 \mathrm{~mm}$, and effective length of $450 \mathrm{~mm}$; applied voltage, $19 \mathrm{kV}$ (operation current, $50 \mu \mathrm{A}$ ); temperature, $25^{\circ} \mathrm{C}$; hydrodynamic injection, height of $25 \mathrm{~mm}$ for $300 \mathrm{~s}$; and detection, $254 \mathrm{~nm}$. Peak assignment: 1, CPr-5'-dGMP; 2, 5'-dAMP; 3, Et-5'-dGMP; 4, 5'dCMP; 5, 5-dTMP; 6, 5'-dGMP; 7, 5'-CMP (IS) (Concentrations of each nucleotides were prepared to be $1 \times 10^{-4} \mathrm{~mol} / \mathrm{L}$ in the sample solution).

to 9.8, as shown in Fig. 2. The electrophoretic mobilities of 5'-dGMP, Et-5'-dGMP, and 5'-dTMP increased remarkably by increasing the $\mathrm{pH}$ because of dissociation of their acidic $\mathrm{NH}$ groups ( $\mathrm{p} K_{\mathrm{a}}$ values are 9.2, unknown, and 9.8, respectively). Those of the other four nucleotides changed slightly.

The present system employed a simple CZE mode in the presence of a fast electroosmotic flow (EOF). Therefore, there was an obvious tendency in the migration orders, i.e., purine base adducts (CPr-Gua and Et-Gua) $>$ authentic purines $>$ authentic pyrimidines, which depended on the size of the nucleotides. A complete separation of the six nucleotides was achieved at $\mathrm{pH}$ 9.67, as shown in Fig. 3. The order of 
Table 1 Calibration curves for quantification of the six nucleotides

\begin{tabular}{lcc}
\hline \multicolumn{1}{c}{ Nucleotide } & Equation & $\begin{array}{c}\text { Correlation } \\
\text { coefficient, } r^{2}\end{array}$ \\
\hline CPr-5'-dGMP & $Y=1.40 \times 10^{5} X+0.0034$ & 0.999 \\
Et-5'-dGMP & $Y=1.69 \times 10^{5} X+0.0307$ & 1.000 \\
$5^{\prime}$-dAMP & $Y=2.10 \times 10^{5} X+0.0569$ & 1.000 \\
5'-dTMP & $Y=1.42 \times 10^{5} X+0.0198$ & 0.999 \\
5'-dCMP & $Y=1.06 \times 10^{5} X+0.0227$ & 0.998 \\
5'-dGMP & $Y=2.18 \times 10^{5} X+0.0435$ & 1.000 \\
\hline
\end{tabular}

$n=9$ each (from $4.19 \times 10^{-5}$ to $\left.4.19 \times 10^{-4}[\mathrm{~mol} / \mathrm{L}]\right) ; Y$, relative values of the integrated peak areas of the UV absorbance of the analytes to those of IS; $X$, the concentrations of the six analytes in sample solutions at injections $[\mathrm{mol} / \mathrm{L}]$.

Et- $5^{\prime}$-dGMP and $5^{\prime}$-dAMP was reversed because the acidic NH protons on the guanine moiety of Et-5'-dGMP would be considerably dissociated at $\mathrm{pH} 9.67$.

Peak broadenings were observed in the analysis of nuclease reaction solutions, including complex matrix components instead of standard solutions of the nucleotides. The broadenings were resolved by the addition of $80 \mathrm{mM}$ SDS to the borate running solution, while negligibly changing the migration behaviors of the seven strongly charged anions, including IS. On the other hand, nucleosides and nucleobases, as possible impurities of minor degradation products, were separated simultaneously using an additional MEKC mode. The nucleobases and the nucleosides were detected much faster than the seven nucleotides, and did not disturb their detections under the experimental condition.

Calibration curves were made for the concentrations of the six nucleotides versus their relative peak areas to those of IS (5'-CMP) under the optimized conditions noted in Fig. 3. The linear equations are summarized in Table 1 . The reproducibility of the migration time of the seven nucleotides, including IS for mixtures of the standard solutions, was from 0.9 to 1.3 as RSD $(\%, n=8)$ as a diurnal variation.

Analysis of hydrolysis reactions from native CT-DNA and damaged ones to constituent 2'-deoxyribonucleoside-5'monophosphates by three endonucleases

In general, enzymes have high substrate selectivities. Native nucleases thus prefer the authentic base moieties in DNAs rather than the damaged ones as substrates to a varied degree. Therefore, it has to be confirmed that the candidate nucleases give quantitatively the object adducts as the corresponding $2^{\prime}$-deoxyribonucleoside-5'-monophosphates. The aim of this work was to find a type-A endonuclease giving Et-5'-dGMP and CPr-5'-dGMP from DNAs quantitatively.

Three commercially available type-A endonucleases, NS1, NP1, and Bal 31, were examined using native CT-DNA. Two kinds of damaged CT-DNAs, including CPr-Gua or Et-Gua, were prepared as mentioned above. As expected, marked differences in the substrate selectivities were observed among the three endonucleases in the hydrolysis of the two damaged DNA. All of the three enzymes hydrolyzed the native CT-DNA almost quantitatively to generate the four authentic 2'-deoxyribonucleoside-5'-monophosphates.

Figures 4 and 5 indicate electropherograms of the damaged CT-DNA samples, including CPr-Gua (CT-DNA-CPr) and Et-Gua (CT-DNA-Et), respectively. The four authentic nucleotides were detected in all electropherograms, as shown in
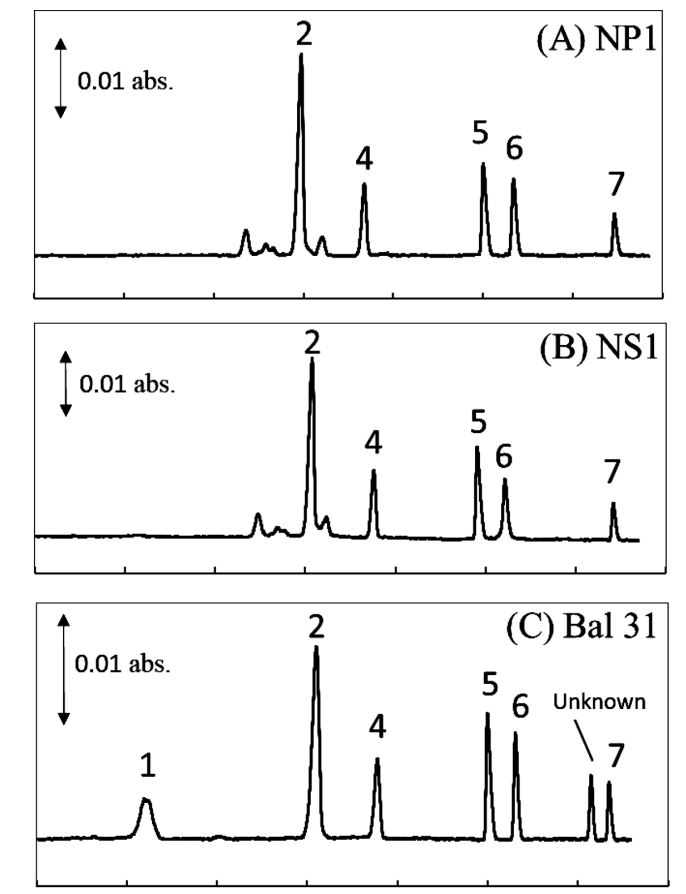

$\begin{array}{llllllll}-1.3 & -1.4 & -1.5 & -1.6 & -1.7 & -1.8 & -1.9 & -2.0\end{array}$

$$
m_{\mathrm{ep}} \times 10^{8} / \mathrm{m}^{2} \mathrm{~V}^{-1} \mathrm{~s}^{-1}
$$

Fig. 4 Electropherograms of the hydrolysis solutions of damaged CT-DNA including CPr-Gua (CT-DNA-CPr) by NP1 (A), NS1 (B), and Bal $31(\mathrm{C})$. CE conditions: same as those in Fig. 3. Peak assignment: 1, CPr-5'-dGMP; 2, 5'-dAMP; 4, 5'-dCMP; 5, 5-dTMP; 6, 5'-dGMP; 7, 5'-CMP (IS).

Figs. 4 and 5. However, CPr-5'-dGMP and Et-5'-dGMP did not appear in NP1 and NS1 hydrolysis, suggesting that NP1 and NS1 did not recognize the moieties of Et-Gua and CPr-Gua in the damaged DNA as their substrates. It was confirmed by spiking the standard that the peaks marked using an asterisk in Fig. 4(b) and Fig. 5(b) were not those of Et- $5^{\prime}$-dGMP. On the other hand, CPr-5'-dGMP and Et-5'-dGMP were observed clearly in Bal 31 hydrolysis.

In Figs. 4 and 5, all electropherograms were redrawn using the electrophoretic mobility $\left(m_{\mathrm{ep}}\right)$ as X-axis instead of migration time $(t)$ (see the Experimental section). The aim was to decrease the variability in $\mathrm{X}$-axis values by omitting the changes in EOF velocity between each run. Thus, peak assignment can be facilitated by a comparison of the electropherograms. Interestingly, there was a strong homology between NP1 hydrolysis (Fig. 4(a) and Fig. 5(a)) and NS1 hydrolysis (Fig. 4(b) and Fig. 5(b)), judging from $m_{\mathrm{ep}}$ of each peak, height ratios, and the shapes of the minor peaks. NP1 and NS1 thus indicated similar substrate selectivities. The minor peaks would be mostly those of intermediate products, such as dinucleotides, including CPr-Gua (Figs. 4(a) and 4(b)) or Et-Gua (Figs. 5(a) and 5(b)). No minor peaks were observed in the case of Bal 31 (Fig. 4(c) and Fig. 5(c)).

NP1 is used as an endonuclease in several kits for determining 8-oxo-guanine in DNAs to hydrolyze them to $2^{\prime}$-deoxyribonucleoside-5'-monophosphates, including 8-oxo-5'-dGMP. Therefore, the famous oxidized adduct moieties in DNA must become a good substrate of NP1.31 On the other hand, Falcone and Box studied the NP1 hydrolysis of di-deoxyribonucleoside monophosphates, including many kinds of damaged bases. 

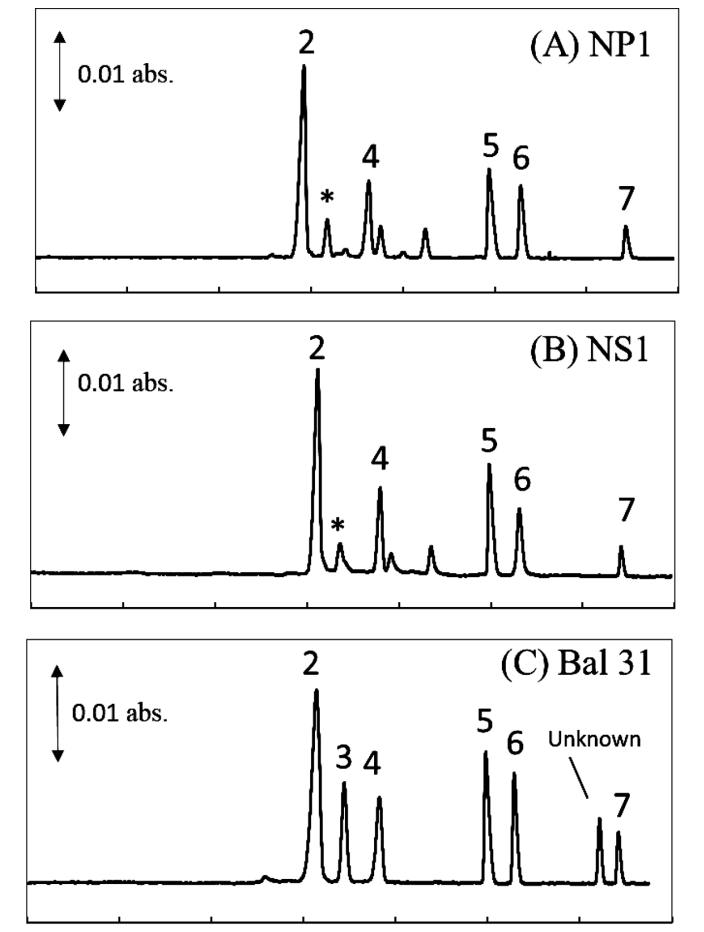

$\begin{array}{llllllll}-1.3 & -1.4 & -1.5 & -1.6 & -1.7 & -1.8 & -1.9 & -2.0\end{array}$

$$
m_{\mathrm{ep}} \times 10^{8} / \mathrm{m}^{2} \mathrm{~V}^{-1} \mathrm{~s}^{-1}
$$

Fig. 5 Electropherograms of the hydrolysis solutions of damaged CT-DNA including Et-Gua (CT-DNA-Et) by NP1 (A), NS1 (B), and Bal 31 (C). CE conditions: same as those in Fig. 3. Peak assignment: 2, 5'-dAMP; 3, Et-5'-dGMP; 4, 5'-dCMP; 5, 5'-dTMP; 6, 5'-dGMP; 7, $5^{\prime}$-CMP (IS).

They indicated the disturbance magnitude by damages in the base moieties against NP1 hydrolysis to generate the corresponding nucleoside monophosphates, depending on the positions and structure of the damages. In general, native nucleases, including NP1, preferred the authentic base moieties as their substrates. The positive selectivity of NP1 toward the 8-oxo-Gua moieties would be an unusual case. NP1 also hydrolyzes 2'-deoxyribonucleoside-3'-monophosphates to the corresponding $2^{\prime}$-deoxyribonucleosides and also has high substrate selectivities toward the authentic base moieties. Because of the substrate selectivity, NP1 is used to improve the sensitivity of a ${ }^{32} \mathrm{P}$-labeling method for detecting DNA adducts. ${ }^{32}$ Vaca et al. detected a much larger amount of Et-Gua from DNAs of a patient having chronic alcoholism compared with those of controls using the improved ${ }^{32} \mathrm{P}$-labeling method. ${ }^{11}$ NP1 should not have dephospholylated Et-3'-dGMP practically. The present result, as shown in Fig. 2, about the damaged CT-DNAs would also be attributed to a similar recognition mechanism with that in the improved ${ }^{32} \mathrm{P}$-labeling method.

Table 2 summarizes the quantitative ratios of each nucleotide generated from native CT-DNA, CT-DNA-Et, and CT-DNA-CPr using NP1, NS1, and Bal 31. The determined concentrations of the six nucleotides in the reaction solutions were divided by those of 5'-dAMP. The ratio of adenine-thymine against Guacytocine in native CT-DNA is known to be 1.000:0.721. ${ }^{33}$ As shown in Table 2, native CT-DNA would hydrolyze almost quantitatively to generate $5^{\prime}$-dAMP, 5'-dCMP, 5'-dGMP, and $5^{\prime}$-dTMP by all of the three type-A endonucleases. It should be
Table 2 Quantitative ratios of the constituent $2^{\prime}$-deoxyribonucleoside- $5^{\prime}$-monophosphate by the nuclease reactions of Calf thymus DNA

\begin{tabular}{clccc}
\hline Nuclease & Nucleotide & CT-DNA & CT-DNA-Et & CT-DNA-CPr \\
\hline Bal31 & 5'-dAMP & 1 & 1 & 1 \\
& 5'-dTMP & 0.98 & 1.00 & 1.00 \\
& 5'-dCTP & 0.76 & 0.75 & 0.76 \\
& 5'-dGMP & 0.75 & 0.40 & 0.40 \\
& Et-5'-dGMP & n.d. & 0.38 & n.d. \\
& CPr-5'-dGMP & n.d. & n.d. & 0.40 \\
NP1 & 5'-dAMP & 1 & 1 & 1 \\
& 5'-dTMP & 0.99 & 0.97 & 1.02 \\
& 5'-dCMP & 0.77 & 0.72 & 0.87 \\
& 5'-dGMP & 0.76 & 0.42 & 0.42 \\
& Et-5'-dGMP & n.d. & n.d. & n.d. \\
& CPr-5'-dGMP & n.d. & n.d. & n.d. \\
NS1 & 5'-dAMP & 1 & 1 & 1 \\
& 5'-dTMP & 0.99 & 0.97 & 1.02 \\
& 5'-dCTP & 0.74 & 0.72 & 0.91 \\
& 5'-dGMP & 0.70 & 0.36 & 0.40 \\
& Et-5'-dGMP & n.d. & n.d. & n.d. \\
& CPr-5'-dGMP & n.d. & n.d. & n.d. \\
\hline
\end{tabular}

n.d.: not detected.

noted that the sum of the ratios for Et-dGMP and dGMP in CT-DNA-Et and that for CPr-dGMP and dGMP in CT-DNA$\mathrm{CPr}$ about reactions by $\mathrm{Bal} 31$ were almost the same in quantity as that of dGMP in native CT-DNA. This suggests that Bal 31 recognized the Et-Gua moieties and the CPr-Gua moieties as its substrates. Bal 31 can also recognize the authentic base moieties and hydrolyze them to generate Et-5'-dGMP and CPr-5'-dGMP quantitatively.

The minor differences of the component ratios between the ideal ones (1.000:1:000:0.721:0.721) and the given data in Table 2 for $5^{\prime}$-dAMP, 5'-dTMP, 5'-dCMP, and 5'-dGMP in native CT-DNA could be attributable to the difficulty of estimating the accurate concentrations of the nucleotides in their standard solutions, as mentioned above. However, the deviations in the cases of NP1 and NS1 for CT-DNA-Et seemed to be relatively large, and Bal 31 would hydrolyze it with reasonable yielding ratios. This may indicate that the reactions were not finished by both NP1 and NS1. Thus, a lower tolerance of NP1 and NS1 against changes in the structures of DNA bases was found compared with Bal 31.

\section{Conclusions}

In the analysis of DNA damages, it is important to evaluate the candidate nucleases from the viewpoint of substrate selectivities and practical enzyme activities to ensure the quantitative yields of object damaged nucleotides. ${ }^{31}$ The present CE method is very useful for determining nucleotide monomers, including damaged ones in nuclease reaction solutions of DNA. Good separations with high resolution are promising by optimization of the running buffer $\mathrm{pH}$ as a simple and convenient separation parameter in relation to the $\mathrm{p} K_{\mathrm{a}}$ values of functional groups in the base moieties of nucleotides. Existence frequencies of EtGua and CPr-Gua in DNA can indicate cancer risks of individual persons related largely to drinking. It was found that Bal 31 was suitable for obtaining the two corresponding $2^{\prime}$-deoxyribonucleoside- $5^{\prime}$-monophosphates as risk markers quantitatively from DNAs. The detection of trace amounts of Et-Gua and 
CPr-Gua in mouse DNAs as Et- $5^{\prime}$-dGMP and CPr-5'-dGMP, respectively, is under study in combination with on-line $\mathrm{CE}$ enrichment methods and the present Bal 31 pretreatment.

\section{Acknowledgements}

The authors thank N. Sakaida and A. Yoshimoto for their support with the experiments. This work was partly supported by JSPS KAKENHI Grant Number 15K05546 (Grant-in-Aid for Scientific Research (C) for Y. E.). This work was also supported in part by Koshiyama Science and Technology Foundation. The authors would like to thank Enago (www.enago.jp) for the English language review.

\section{References}

1. S. Balbo, S. S. Hecht, P. Upalyaya, and P. W. Villata, Anal. Chem., 2014, 86, 1744.

2. R. Kanaly, S. Matsui, T. Hanaoka, and T. Matsuda, Mutat. Res., 2007, 625, 83 .

3. X. Li, L. Liu, W. J. Chen, B. Zhu, H. Chen, H. Hou, and Q. Hu, J. Chromatogr. B, 2017, 1060, 451.

4. S. Inagaki, Y. Esaka, Y. Deyashiki, M. Sako, and M. Goto, J. Chromatogr. A, 2003, 987, 341.

5. T. Matsuda, Carcinogenesis, 2007, 28, 2363.

6. T. Matsuda, H. Yabushita, R. Kanaly, S. Shibutani, and A. Yokoyama, Chem. Res. Toxicol., 2006, 19, 1374.

7. H. Murakami, R. Horiba, T. Iwata, Y. Miki, B. Uno, T. Sakai, K. Kaneko, Y. Ishihama, N. Teshima, and Y. Esaka, Talanta, 2018, 177, 12.

8. I. Terashima, N. Suzuki, and S. Shibutani, Chem. Res. Toxicol., 2002, 15, 305.

9. S. Shibutani, S. Y. Kim, and N. Suzuki, Methods Mol. Biol., 2006, 314, 307.

10. Y. Esaka, S. Inagaki, and M. Goto, J. Chromatogr. B, 2003, 797, 321.

11. J.-L. Fang and C. E. Vaca, Carcinogenesis, 1997, 18, 627.

12. S. Inagaki, Y. Esaka, M. Sako, and M. Goto, Electrophoresis, 2001, 22, 3408.

13. J. M. Falcone and H. C. Box, Biochim. Biophys. Acta, 1997, 1337, 267.

14. S. Inagaki, Y. Esaka, M. Goto, Y. Deyashiki, and M. Sako,
Biol. Pharm. Bull., 2004, 27, 273.

15. P. J. Brooks, M.-A. Enoch, D. Gordon, T.-K. Li, and A. Yokoyama, PLoS Medicine, 2009, 6, 0258.

16. Internal Agency for Research on Cancer, IARC Monographs on the Evaluation of Carcinogenic Risks to Human. Alcohol Consumption and Ethyl Carbamate, 2010, 96, 41.

17. S. S. Hecht, Chem. Res. Toxicol., 2017, 30, 367.

18. C. C. Garcia, F. P. Freitas, A. B. Sanchez, P. Di Mascio, and M. H. Medeiros, Chem. Res. Toxicol., 2013, 26, 1602.

19. C. C. Garcia, J. P. Angeli, F. P. Freitas, O. F. Gomes, T. F. de Oliveira, A. P. Loureiro, P. Di Mascio, and M. H. Medeiros, J. Am. Chem. Soc., 2011, 133, 9140.

20. T. Tsuda, G. Nakagawa, M. Sato, and K. Yagi, J. Appl. Biochem., 1983, 5, 330.

21. K. H. Row, W. H. Griest, and M. P. Maskarinec, J. Chromatogr., 1987, 409, 193.

22. C. W. Demarest, E. A. Monnot-Chase, J. Jiu, and R. Weinberger, "Capillary Electrophoresis, Theory and Practice", ed. P. D. Grossman and C. P. Colburn, 1992, Chap. 11, Academic Press, 301.

23. A. S. Cohen, S. Terabe, J. A. Smith, and B. L. Karger, Anal. Chem., 1987, 59, 1021.

24. Y. Asakawa, N. Tokida, C. Ozawa, M. Ishiba, O. Tagaya, and N. Asakawa, J. Chromatogr. A, 2008, 1198-1199, 80.

25. M. Sako, H. Kawada, and K. Hirota, J. Org. Chem., 1999, 64, 5719.

26. M. Sako and I. Yaekura, Tetrahedron, 2002, 58, 8413.

27. Y. Nishimura, T. Fukao, M. Sugimoto, H. Sawada, M. Itoh, and M. Nanba, Shokuhin Kogyo Gakkaishi (Journal of Food Science and Technology, in Japanese), 1990, 37, 687.

28. M. R. Green and J. Sambrook, "Molecular Cloning, A Laboratory Manual", 4th ed., 2012, Chap. 4, Cold Spring Harbor Laboratory Press, 79.

29. P. D. Grossman "Capillary Electrophoresis, Theory and Practice", ed. P. D. Grossman and C. P. Colburn, 1992, Chap. 4, Academic Press, 111.

30. Y. Esaka, S. Inagaki, M. Goto, and M. Sako, Electrophoresis, 2001, 22, 104.

31. X. Huang, J. Powell, L. A. Mooney, C. Li, and K. Frenkel, Free Radic Biol Med., 2001, 31, 1341.

32. J. Marmur and P. Doty, J. Mol. Biol., 1962, 5, 109.

33. M. V. Reddy and K. Randerath, Carcinogenesis., 1986, 7, 1543. 